\title{
Upregulation of mitochondrial alternative oxidase pathway protects photosynthetic apparatus against photodamage under chilling stress in Rumex K-1 leaves
}

\author{
D. CHENG ${ }^{*, * *}, \mathrm{H} \mathrm{GAO}^{* *}$, and L. ZHANG ${ }^{* *,+}$ \\ College of Landscape Architecture and Forestry, Qingdao Agricultural University, Qingdao, Shandong, China* \\ State Key Laboratory of Crop Biology, College of Life Sciences, Shandong Agricultural University, Tai'an, \\ Shandong, China ${ }^{* *}$ \\ Institute of Oceanology, Chinese Academy of Sciences, Qingdao, Shandong, China ${ }^{* * *}$
}

\begin{abstract}
This study aimed to clarify the role of mitochondrial alternative oxidase (AOX) pathway upregulation in photoprotection under chilling stress in Rumex K-1 leaves. Malate-oxaloacetate shuttle's activity and AOX pathway's capacity were synchronously enhanced with light intensity increase both in control and chilling-treated leaves. Besides, the AOX pathway was upregulated more significantly when irradiated by light in chilling-treated leaves than that of control. These results suggested that excess reducing equivalents generated by photosynthesis were exported from chloroplasts and oxidized by the AOX pathway at a potentially higher rate in chilling-treated leaves than in control, which avoided the overaccumulation of excess reducing equivalents under light conditions. Also, inhibition of the AOX pathway led to more severe accumulation of hydrogen peroxide in chilling-treated leaves than that in control. Therefore, the mitochondrial AOX pathway was specifically upregulated and employed for sinking excess reducing equivalents produced by photosynthesis, which protected photosynthetic apparatus from photodamage under chilling stress in Rumex K-1 leaves.
\end{abstract}

Keywords: abiotic stress; photosynthesis; respiratory metabolism.

\section{Introduction}

Respiration is a central metabolism vital to cell function. Metabolites produced by respiration (e.g., ascorbic acid and ATP) are indispensable in many synthesizing procedures, being vital to cell growth and maintenance (Juszczuk and Ostaszewska 2011). The electron in mitochondria of higher plants can be transported via the paths of the cyanide-insensitive AOX pathway and cyanide-sensitive cytochrome oxidase (COX) pathway (Raghavendra and Padmasree 2003). By generating proton gradient, the COX pathway accomplish most of the ATP production in the mitochondrion, however, the AOX pathway is nonphosphorylating and capable of oxidizing reducing equivalents in an efficient manner while not getting limited by cellular ATP/ADP ratio or proton gradients across the internal membrane of the mitochondrion (Yoshida et al. 2007, Zhang et al. 2012a).

Photosystem II (PSII) photoinhibition refers to the light-induced loss of PSII electron-transfer activity; this phenomenon is due to the imbalance between the rate of photodamage to PSII and the rate of the repair of damaged PSII (Nishiyama et al. 2006). According to the 'classical' photoinhibition mechanism, excess excitation light energy gives rise to reactive oxygen species (ROS) production, leading to PSII photochemical reaction center inactivation directly. In comparison, Murata et al. (2007) proposed a novel scheme, in which photodamage is initiated by the direct effect of light on the oxygen-evolving complex and ROS suppresses photodamaged PSII repairment largely by inhibiting D1 proteins synthesis de novo. In chloroplast, the use of energy and photosynthetic light absorption should be balanced to avoid ROS from being formed. Under chilling stress, $\mathrm{CO}_{2}$ assimilation is suppressed whilst the excess excitation and reducing equivalents may increase (Höglind et al. 2011). Besides, ROS accumulation has often been observed in chloroplasts due to the deactivation of ROS-scavenging enzymes at low temperatures, which was considered as the primary cause of PSII photoinhibition (Zhang et al. 2011a).

In the past two decades, the mutual effects of photosynthesis and respiration arouse growing attention (Ogawa and Sonoike 2015). Some vital metabolic pathways (e.g., nitrogen assimilation and photorespiration) need reactions taking place in chloroplasts and mitochondria (Betti et al. 2016). It also has been presented that excessive reducing

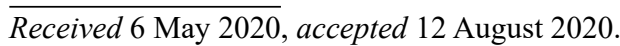

+Corresponding author: e-mail: 819296624@qq.com, 8562034@qq.com

Abbreviations: AOX - alternative oxidase; COX - cytochrome oxidase; $\mathrm{F}_{\mathrm{v}} / \mathrm{F}_{\mathrm{m}}$ - maximal quantum yield of PSII; NADP-MDH NADP-malate dehydrogenase; OAA - oxaloacetate; ROS - reactive oxygen species; SHAM - salicylhydroxamic acid; $\varphi_{\mathrm{Eo}}-$ the quantum yield of electron transport; $\Psi_{0}$ - the excitation efficiency for electron transport beyond $\mathrm{Q}_{\mathrm{A}}$.

Acknowledgments: State Key Laboratory of Crop Biology (2017KF05) financed this study. 
equivalents generated as NADPH by photosynthesis could be transported to mitochondria through shuttle machinery (e.g., malate-OAA shuttle) and oxidated by respiratory electron transport chain (Noguchi and Yoshida 2008). Several studies have demonstrated that photosynthetic evolution rate and PSII photochemical efficiency decreased obviously when the AOX pathway was inhibited in leaves (Zhang et al. 2012a,b) or protoplasts (Dinakar et al. 2010a,b). Also, the AOX pathway was induced to oxidize excess reducing equivalents generated by photosynthesis in Arabidopsis thaliana (Yoshida et al. 2007). AOX pathway was reported to be vital in regulating cellular redox and ROS homeostasis to optimize photosynthesis during restriction of the cytochrome oxidase pathway in Arabidopsis thaliana (Vishwakarma et al. 2015). The AOX pathway was also important in optimizing photosynthesis under suboptimal temperatures stress and hyperosmotic stress in pea plants (Dinakar et al. 2016). The significance of the AOX pathway in optimizing photosynthesis under elevated $\mathrm{CO}_{2}$, de-etiolation, high light, drought, and other stresses in plants and algae is also well explained (Vanlerberghe 2013, Vishwakarma et al. 2015, Dahal and Vanlerberghe 2017, Hu et al. 2018, Murik et al. 2019, Sunil et al. 2019). However, whether the mitochondrial AOX pathway is also critical to the photoprotection of plants illuminated under chilling stress requires further elucidation. The present study aims to verify the speculation that under chilling stress, the AOX pathway in the mitochondrion is upregulated, and it is adopted as an extra-chloroplastic sink for excess reducing equivalents generated by photosynthesis, preventing photosynthetic electron transport chain from being reduced overly and then protecting photosynthetic apparatus from being photodamaged in Rumex K-1 leaves.

\section{Materials and methods}

Plant material and treatments: This study was conducted in a growth cabinet of Shandong Agriculture University $\left(36^{\circ} 11^{\prime} \mathrm{N}, 117^{\circ} 7^{\prime} \mathrm{E}\right)$. Rumex $\mathrm{K}-1$ (a hybrid of Rumexpatientia $\times R$. tianschaious), is a forage crop exhibiting leaf protein in high contents. Rumex K-1 plants grew at $22 / 18^{\circ} \mathrm{C}$ (day/ night) in a 14-h photoperiod [500 $\mu$ mol(photon) $\left.\left.\mathrm{m}^{-2} \mathrm{~s}^{-1}\right)\right]$ in a pot $(20 \mathrm{~cm}$ in diameter, $30 \mathrm{~cm}$ in height) containing soil. Four-week-old plants were used in this study. For avoiding any possible drought and nutrient stresses, nutrients and water were offered sufficiently.

Three Rumex K-1 plants were used to obtain 20 leaf discs $(0.8-\mathrm{cm}$ diameter) for experiments, ten leaf discs were punched from each functional leaf of the three plants symmetrically along the vein, then 30 discs were mixed and 20 discs were chosen to use randomly. One group (20 discs) of Rumex K-1 leaf discs, adaxial side up, were treated as control $\left(25^{\circ} \mathrm{C}\right)$ or chilling stress $\left(10^{\circ} \mathrm{C}\right)$ for $6 \mathrm{~h}$ in an electric-heated thermostatic water bath under different light intensities $[0,100,300,500$ or $800 \mu \mathrm{mol}($ photon) $\mathrm{m}^{-2} \mathrm{~s}^{-1}$. Another group (20 discs) was infiltrated with or without (control) salicylhydroxamic acid (SHAM, $1 \mathrm{mM}$ ) solution, respectively. Subsequently, the discs were exposed to control $\left(25^{\circ} \mathrm{C}\right)$ or chilling stress $\left(10^{\circ} \mathrm{C}\right)$ for $6 \mathrm{~h}$ under low light $\left[300 \mu \mathrm{mol}\left(\right.\right.$ photon) $\mathrm{m}^{-2} \mathrm{~s}^{-1}$ ] or high light [800 $\mu$ mol(photon) $\mathrm{m}^{-2} \mathrm{~s}^{-1}$. At least five measurements were made in each experiment in this study.

Respiratory rate: The respiratory rates in leaf discs were measured using an Oxytherm oxygen electrode (Hansatech Instruments, Norfolk, UK) at $25^{\circ} \mathrm{C}$ according to Yoshida et al. (2006). Before respiratory measurements, we incubated the leaf discs in dark for $10 \mathrm{~min}$. For inhibiting the AOX or COX pathway, respectively, SHAM $(20 \mathrm{mM})$ or KCN $(10 \mathrm{mM})$ was employed. The percentage of the activity of cytochrome respiration, alternative respiration, and nonspecific respiration was $84.1,15.8$, and $2.6 \%$, respectively, in untreated Rumex $\mathrm{K}-1$ leaves, so the nonspecific respiration was negligible and the AOX pathway capacity was defined as $\mathrm{O}_{2}$ uptake rate in the presence of $\mathrm{KCN}$, the COX pathway capacity was defined as $\mathrm{O}_{2}$ uptake rate in the presence of SHAM, total respiration was defined as $\mathrm{O}_{2}$ uptake rate without any inhibitor in this study.

NADP-malate dehydrogenase (NADP-MDH) activity: NADP-MDH (EC 1.1.1.82) was extracted and assayed according to Dutilleul et al. (2003) with some modifications. Leaf discs $\left(7.5 \mathrm{~cm}^{2}\right)$ were ground with a mortar and pestle in liquid nitrogen and then added into $50 \mathrm{mM}$ 4-(2-hydroxyethyl)-1-piperazine ethane sulphonic acid- $\mathrm{KOH}(\mathrm{pH} 7.5)$ buffer that contained $0.05 \%(\mathrm{v} / \mathrm{v})$ Triton $X-100,5 \%(\mathrm{w} / \mathrm{v})$ insoluble polyvinylpyrrolidone, a protease inhibitor tablet, $5 \mathrm{mM}$ dithiothreitol (DTT), $1 \mathrm{mM} \mathrm{Na} \mathrm{NDDTA}_{2}$, and $10 \mathrm{mM} \mathrm{MgCl}$. After the buffer was centrifugated for $5 \mathrm{~min}$ at $14,000 \times g$, the enzyme activity in the supernatant was measured with a $U V-2550$ spectrophotometer (Shimadzu, Kyoto, Japan). The initial NADP-MDH activity was measured directly in the supernatant. To fully activate the enzyme, $0.2 \mathrm{~mL}$ of supernatant was preincubated for $30 \mathrm{~min}$ at $25^{\circ} \mathrm{C}$ in $120 \mathrm{mM} \mathrm{KCl}$, $100 \mathrm{mM}$ DTT, $0.4 \mathrm{mM} \mathrm{Na}{ }_{2}$ EDTA, $0.0025 \%$ (v/v) Triton $X-100$, and $40 \mathrm{mM}$ Tricine- $\mathrm{KOH}$ (pH 9.0). Assays were conducted in $2 \mathrm{mM}$ OAA, $0.2 \mathrm{mM}$ NADPH, $5 \mathrm{mM}$ DTT, $1 \mathrm{mM} \mathrm{Na} \mathrm{EDTA}_{2}, 150 \mathrm{mM} \mathrm{KCl}$, and $40 \mathrm{mM}$ Tricine-KOH ( $\mathrm{pH} 8.3$ ), plus the sample.

Chlorophyll (Chl) $\boldsymbol{a}$ fluorescence transient: The Chl $a$ fluorescence (OJIP) transient was measured using a Handy Plant Efficiency Analyser (Hansatech Instruments, Norfolk, UK). OJIP transients were analyzed by the JIP-test (Strasser and Strasser 1995, Strasser et al. 2000), using the following original data: (1) the fluorescence intensity at $20 \mathrm{~s}$, considered to be $\mathrm{F}_{\mathrm{o}}$ when all PSII reaction centers were open; (2) the maximal fluorescence intensity, $\mathrm{F}_{\mathrm{m}}\left(\mathrm{F}_{\mathrm{P}}\right.$, at about $\left.300 \mathrm{~ms}\right)$, assuming that the excitation intensity was high enough to close all of the PSII reaction centers; and (3) the fluorescence intensities at $2 \mathrm{~ms}$ (J-step, $\mathrm{F}_{\mathrm{J}}$ ). To quantify PSII behavior referring to time 0 , the following parameters were employed: (1) the maximal quantum yield of PSII $\left(\mathrm{F}_{\mathrm{v}} / \mathrm{F}_{\mathrm{m}}\right), \mathrm{F}_{\mathrm{v}} / \mathrm{F}_{\mathrm{m}}=1-\left(\mathrm{F}_{\mathrm{o}} / \mathrm{F}_{\mathrm{m}}\right)$; (2) the relative variable fluorescence at J-step $\left(\mathrm{V}_{\mathrm{J}}\right)$, 
$\mathrm{V}_{\mathrm{J}}=\left(\mathrm{F}_{\mathrm{J}}-\mathrm{F}_{\mathrm{o}}\right) /\left(\mathrm{F}_{\mathrm{m}}-\mathrm{F}_{\mathrm{o}}\right)$; (3) the specific energy fluxes (per reaction center) for absorption (ABS/RC), trapping $\left(\mathrm{TR}_{0} / \mathrm{RC}\right)$, and electron transport $\left(\mathrm{ET}_{0} / \mathrm{RC}\right) ;(4)$ the efficiency with which a trapped exciton can move an electron into the electron transport chain further than $\mathrm{Q}_{\mathrm{A}}$ (the excitation efficiency for electron transport beyond $\mathrm{Q}_{\mathrm{A}}$, $\Psi_{0}=\mathrm{ET}_{0} / \mathrm{TR}_{0}$ ) and the quantum yield of electron transport $\left(\varphi_{\mathrm{Eo}}=\mathrm{ET}_{0} / \mathrm{ABS}\right)$.

Hydrogen peroxide content: The leaf discs $\left(7.5 \mathrm{~cm}^{2}\right)$ were ground in liquid nitrogen and extracted with $80 \%$ acetone, then the solution was centrifugated at $16,000 \times g$ for $10 \mathrm{~min}$, the supernatant $(1.3 \mathrm{ml})$ was neutralized to pH 8.5 with $17 \mathrm{M} \mathrm{NH}_{4} \mathrm{OH}$, and then used immediately to assay $\mathrm{H}_{2} \mathrm{O}_{2}$ content by monitoring the $\mathrm{A}_{410}$ of a titaniumperoxide complex with a $U V-2550$ spectrophotometer (Shimadzu, Kyoto, Japan) according to the method of Patterson et al. (1984).

Chemicals: All the chemicals used in this study were manufactured by Sigma.

Statistical analysis: The results were presented as the means \pm SE of at least three independent measurements. A least significant difference (LSD) was used to analyze differences between the measurements and Tukey's honestly significant difference (HSD) was used for the multiple comparisons at a $5 \%$ level of significance using SPSS 16.0 software (SPSS Inc., Chicago, IL, USA).

\section{Results}

Effects of chilling stress on respiratory rates: Compared with control leaves, total respiratory rates were downregulated (Fig. 1A), COX pathway capacities decreased significantly (Fig. $1 B$ ) but AOX pathway capacities increased (Fig. 1C,D) in chilling-treated leaves at various light intensities. Accordingly, the downregulation of total respiratory rates occurred due to the downregulation in the capacity of the COX pathway in chilling-treated leaves at various light intensities. Besides, both in control and chilling-treated leaves, total respiratory rate and AOX pathway capacity increased gradually (Fig. $1 A, C$ ), whereas COX pathway capacity scarcely altered (Fig. $1 B$ ) with the rise in light intensity. Thus, the gradual increase in total respiratory rate along with the light intensity was due to the rise in AOX pathway respiratory rate both in control and chilling-treated leaves.

Effects of chilling stress on characteristics of NADPMDH: NADP-MDH is a critical enzyme in the malate-OAA shuttle, the primary machinery of reducing equivalents transportation from chloroplasts to mitochondria (Yoshida et al. 2007, Zhang et al. 2011b). NADP-MDH overall activities were not affected by chilling and light treatment (Fig. 2B). NADP-MDH initial activity and activation state were upregulated obviously after light treatment both in control and chilling-treated leaves (Fig. 2A,C), suggesting that excessive reducing equivalents generated by photosynthesis were exported from chloroplasts in the

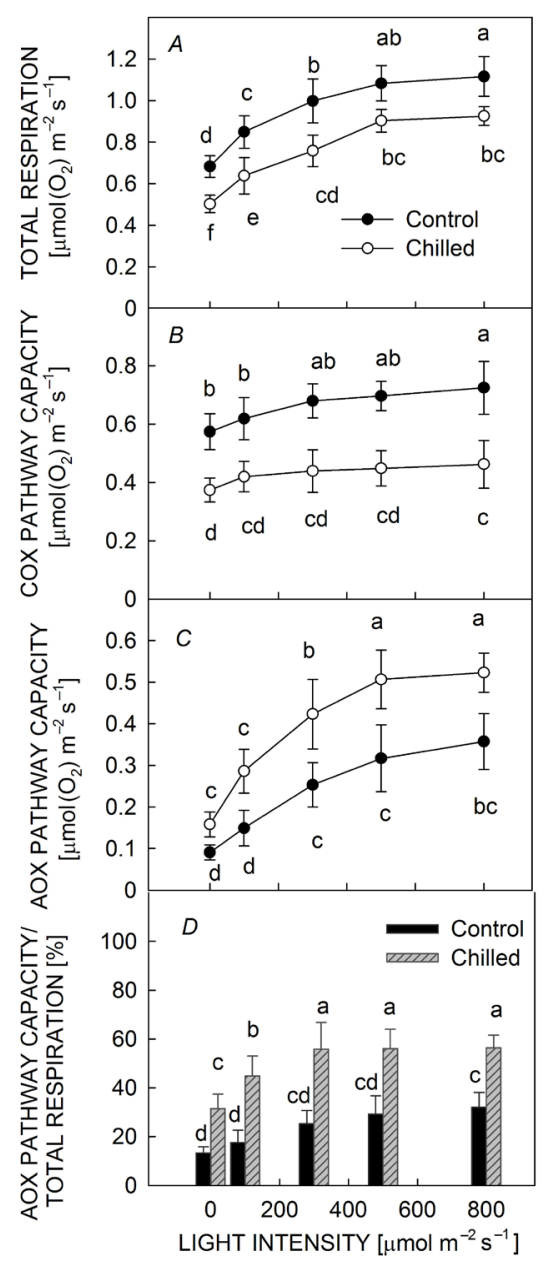

Fig. 1. Effects of chilling stress on total respiratory rates $(A)$, cytochrome oxidase $(\mathrm{COX})$ pathway capacities $(B)$, alternative oxidase (AOX) pathway capacities $(C)$, and AOX pathway capacities/total respiration $(D)$ at different light intensities in Rumex K-1 leaves. Rumex K-1 leaf discs were exposed to control $\left(25^{\circ} \mathrm{C}\right)$ or chilling stress $\left(10^{\circ} \mathrm{C}\right)$ conditions and different light intensities $\left[0,100,300,500\right.$, or $800 \mu \mathrm{mol}$ (photon) $\left.\mathrm{m}^{-2} \mathrm{~s}^{-1}\right]$ for $6 \mathrm{~h}$. Means \pm SE of five replicates are presented. Different letters represent a significant difference between the control and chilled treatment at different light intensities at $P<0.05$, LSD.

light. Chilling treatment did not vary the initial activity and activation state of NADP-MDH before light treatment. However, the NADP-MDH activation state and initial activity were upregulated significantly in chilling-treated leaves compared with control leaves even in the low light (Fig. 2A,C). In the meantime, the rise in NADP-MDH initial activity was synchronized with the enhancement of AOX pathway capacity both in control and chilling-treated leaves as irradiated by the light (Fig. 3).

Effects of SHAM on $\mathbf{F}_{\mathbf{v}} / \mathbf{F}_{\mathbf{m}}, \boldsymbol{\Psi}_{\mathbf{0}}$, and $\boldsymbol{\varphi}_{\mathrm{E} \mathbf{0}}$ : SHAM is a well-known inhibitor of the mitochondrial AOX pathway. It is extensively applied to study the interaction between chloroplasts and mitochondria in both intact tissues and mesophyll protoplasts (Ara et al. 2016). In this study, to 


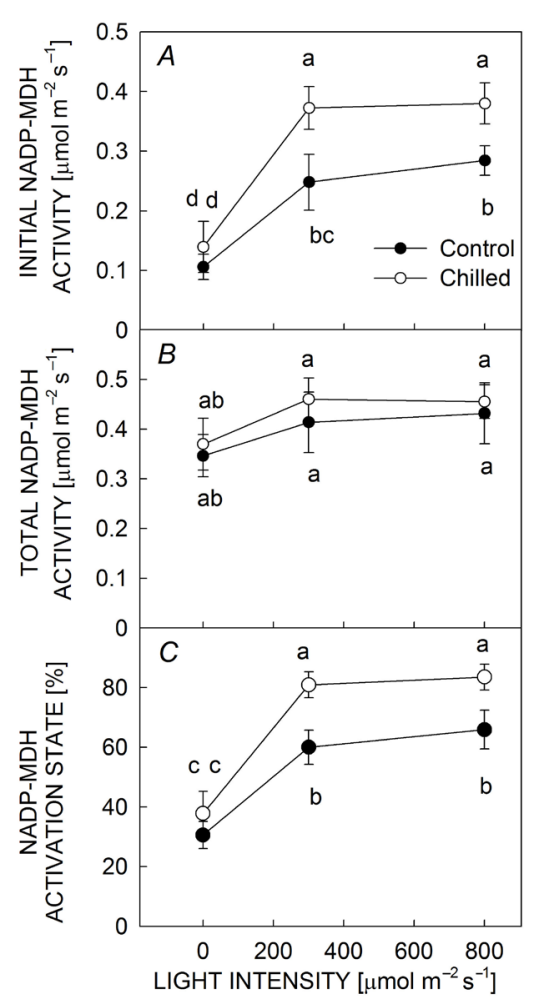

Fig. 2. Effects of chilling stress on characteristics of NADPmalate dehydrogenase (NADP-MDH) at different light intensities in Rumex $\mathrm{K}-1$ leaves. Rumex $\mathrm{K}-1$ leaf discs were exposed to control $\left(25^{\circ} \mathrm{C}\right)$ or chilling stress $\left(10^{\circ} \mathrm{C}\right)$ conditions and different light intensities $\left[0,300\right.$, or $800 \mu$ mol(photon) $\mathrm{m}^{-2} \mathrm{~s}^{-1}$ ] for $6 \mathrm{~h}$. The initial NADP-MDH activity $(A)$ was measured directly in the sample. The total NADP-MDH activity $(B)$ was measured after the full activation by DTT treatment. The NADP-MDH activation state $(C)$ is denoted as initial/total activity $\times 100$. Means \pm SE of five replicates are presented. Different letters represent a significant difference between the control and chilled treatment at different light intensities at $P<0.05$, Tukey's HSD.

further examine the function of the AOX pathway under chilling stress, $1 \mathrm{mM}$ SHAM was employed for AOX pathway inhibition. According to the results, $\mathrm{F}_{\mathrm{v}} / \mathrm{F}_{\mathrm{m}}, \Psi_{0}$, and $\varphi_{\text {Eo }}$ decreased more significantly in chilling-treated leaves compared with control leaves both under low light and high light when the AOX pathway was inhibited (Fig. 4). In our previous study, the fact that $1 \mathrm{mM} \mathrm{SHAM}$ did not affect the actual photochemical efficiency of PSII $\left(\Phi_{\text {PSII }}\right)$ and the photochemical quenching coefficient $\left(\mathrm{q}_{\mathrm{P}}\right)$ in isolated intact Rumex $\mathrm{K}-1$ chloroplasts (Zhang et al. 2011b, Zhang et al. 2012a) demonstrates that $1 \mathrm{mM}$ SHAM had no direct effects on photosynthesis, evidenced by Yoshida et al. (2006) as well. Thus, the observed effects of SHAM treatment on photosynthesis under light were primarily due to mitochondrial AOX pathway inhibition.

Effects of SHAM on $\mathrm{H}_{2} \mathrm{O}_{2}$ content: To examine the effect of inhibition of the AOX pathway on photooxidative damage of PSII, $\mathrm{H}_{2} \mathrm{O}_{2}$ content was measured under low light and high light in Rumex $\mathrm{K}-1$ leaves when the AOX pathway was inhibited. The content of $\mathrm{H}_{2} \mathrm{O}_{2}$ increased

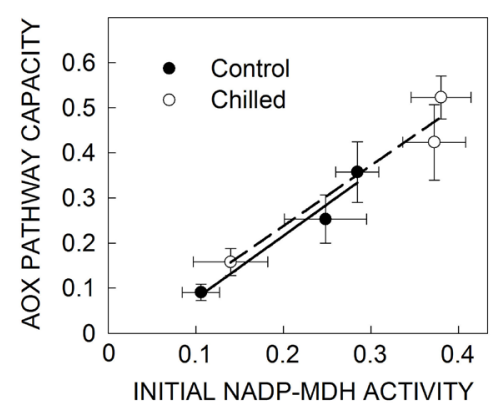

Fig. 3. Relationship between NADP-MDH initial activity and AOX pathway capacity in control and chilling-treated Rumex $\mathrm{K}-1$ leaves at different light intensities $(0,300$, or $800 \mu \mathrm{mol}$ $\left.\mathrm{m}^{-2} \mathrm{~s}^{-1}\right)$. Means $\pm \mathrm{SE}$ of five replicates are presented.

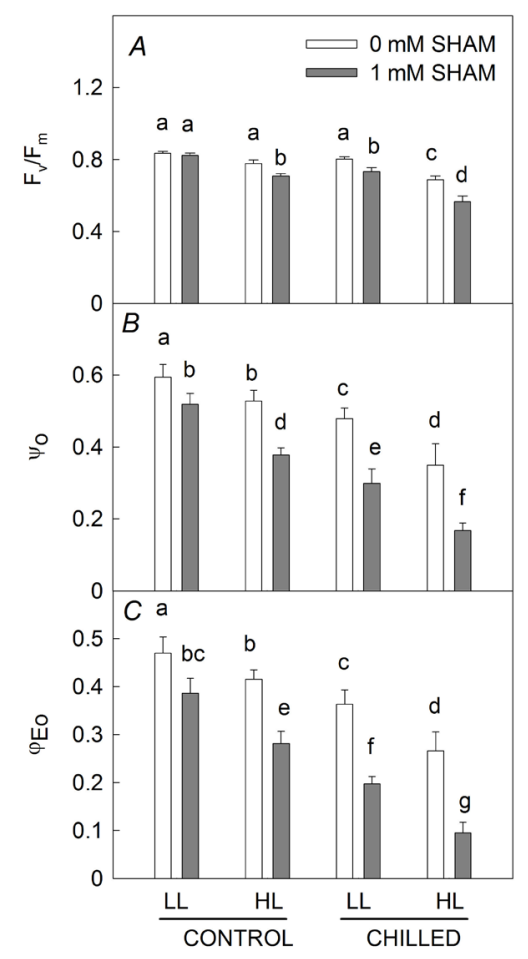

Fig. 4. Effects of salicylhydroxamic acid (SHAM) treatment on the maximal quantum yield of PSII $\left(\mathrm{F}_{\mathrm{v}} / \mathrm{F}_{\mathrm{m}}\right)(A)$, the excitation efficiency for electron transport beyond $\mathrm{Q}_{\mathrm{A}}\left(\Psi_{0}\right)(B)$, and the quantum yield of electron transport $\left(\varphi_{\mathrm{Eo}}\right)(C)$ in control and chilling-treated Rumex K-1 leaves in low light or high light. Rumex K-1 leaf discs were infiltrated with 0 or 1 mM SHAM for $2 \mathrm{~h}$ in dark and then exposed to control $\left(25^{\circ} \mathrm{C}\right)$ or chilling stress $\left(10^{\circ} \mathrm{C}\right)$ conditions and low light $\left[300 \mu\right.$ mol(photon) $\left.\mathrm{m}^{-2} \mathrm{~s}^{-1}\right]$ or high light $\left[800 \mu \mathrm{mol}\left(\right.\right.$ phototn) $\left.\mathrm{m}^{-2} \mathrm{~s}^{-1}\right]$ for $6 \mathrm{~h}$. Means $\pm \mathrm{SE}$ of five replicates are presented. Different letters represent a significant difference between the control and chilled treatment under the low and high light at $P<0.05$, Tukey's HSD.

more significantly in chilling-treated leaves compared with control leaves both under low light and high light when the AOX pathway was inhibited (Fig. 5), suggesting that the photoprotection role of the AOX pathway was more significant to chilling-treated leaves than controls. 


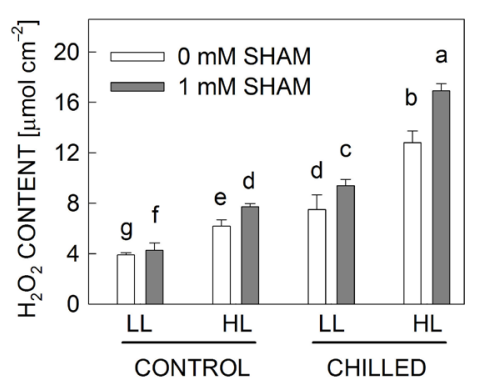

Fig. 5. Effects of salicylhydroxamic acid (SHAM) treatment on hydrogen peroxide $\left(\mathrm{H}_{2} \mathrm{O}_{2}\right)$ content in control and chilling-treated Rumex K-1 leaves in low light or high light. Rumex K-1 leaf discs were infiltrated with 0 or $1 \mathrm{mM}$ SHAM for $2 \mathrm{~h}$ in dark and then exposed to control $\left(25^{\circ} \mathrm{C}\right)$ or chilling stress $\left(10^{\circ} \mathrm{C}\right)$ and low light $[300 \mu \mathrm{mol}$ (photon) $\mathrm{m}^{-2} \mathrm{~s}^{-1}$ ] or high light [800 $\mu \mathrm{mol}\left(\right.$ photon) $\left.\mathrm{m}^{-2} \mathrm{~s}^{-1}\right]$ for $6 \mathrm{~h}$. Means \pm SE of five replicates are presented. Different letters represent a significant difference between the control and chilled treatment under the low and high light at $P<0.05$, Tukey's HSD.

\section{Discussion}

Under chilling stress, $\mathrm{CO}_{2}$ assimilation is suppressed whilst the excessive excitation and reducing equivalents may increase (Höglind et al. 2011). Given this, induction of alternative reducing equivalent sinks will be required to avoid photosynthetic electron transport chain from being reduced overly. It has been suggested that excess reducing equivalents produced by photosynthesis could be transported to mitochondria through malate-OAA shuttle and oxidized by respiratory electron transport chain (Noguchi and Yoshida 2008, Zhang et al. 2012a). NADP-MDH is the key enzyme in the malate-OAA shuttle and its activity is modulated by light energy via the Fd-thioredoxin system (Yoshida et al. 2007, Scheibe and Dietz 2012, Wang et al. 2016). The observation that initial activity and activation state of NADP-MDH increased significantly both under low light and high light after chilling treatment in Rumex K-1 leaves (Fig. 2A,C) indicated that malate-OAA shuttle in chilling-treated leaves was activated more severely by the $\mathrm{Fd}$-thioredoxin system compared with control leaves even under the low light. These results also revealed that the chilling-treated leaves exported excess reducing equivalents from chloroplasts at a potentially higher rate as the electron acceptance by PSI downstream was limited because of suppression of $\mathrm{CO}_{2}$ assimilation, avoiding excess reducing equivalents from accumulating in the stroma under the light. NADP$\mathrm{MDH}$ initial activities and activation state also increased after light treatment in control leaves (Fig. 2A,C), which indicated that, even in control leaves, the exportation of excess reducing equivalents was essential under light conditions.

Generally, these excess reducing equivalents exported from chloroplasts could undergo oxidization by respiratory electron transport chain via internal or external $\mathrm{NAD}(\mathrm{P}) \mathrm{H}$ dehydrogenase located on inner envelope membranes of mitochondria (Noguchi and Yoshida 2008). Which electron transport pathway in mitochondria (COX or AOX pathway) did play the major role in dissipation of chloroplast-derived reducing equivalents? AOX pathway capacity increased (Fig. 1C), whereas COX pathway capacity did not vary (Fig. $1 B$ ) with the rise in light intensity both in control and chilling-treated leaves, suggesting that the AOX pathway instead of COX pathway is vital to chloroplast-derived reducing equivalents dissipation in Rumex K-1 under chilling stress. In the meantime, the fact that coordinated upregulation between AOX pathway capacity and malate-OAA shuttle activity with the rise in light intensity both in control and chilling-treated leaves (Fig. 3) further strengthened the perspective that the AOX pathway functioned as an extra-chloroplastic sink for excess reducing equivalents produced by photosynthesis in Rumex K-1 under chilling stress. Compared with control leaves, the AOX pathway was significantly upregulated (Fig. 1C), whereas COX pathway capacity decreased (Fig. $1 B$ ) in chilling-treated leaves at various light intensities, implying that dissipation of chloroplast-derived reducing equivalents by the $\mathrm{AOX}$ pathway is more vital to chilling-treated leaves than controls.

SHAM (1 mM ) was employed for inhibiting the AOX pathway here to further examine the AOX pathway function in the photoprotection of photosynthetic apparatus under chilling stress. The content of $\mathrm{H}_{2} \mathrm{O}_{2}$ increased more severely when the AOX pathway was suppressed in chilling-treated leaves compared with controls both in the case of low light and high light (Fig. 5), suggesting that the AOX pathway was more important to chilling-treated leaves than control leaves in protecting photosynthetic apparatus from photooxidative damage. Besides, it has been reported that ROS can inactivate the repair of the photodamaged PSII by the suppression of the de novo synthesis of the D1 protein (Nishiyama et al. 2011), which limits the photosynthetic electron transport rate in photosynthesis. In conclusion, the AOX pathway was upregulated and acted as an extra-chloroplastic sink for excess reducing equivalents generated by photosynthesis in Rumex K-1 leaves experiencing chilling stress. An inhibition of the AOX pathway caused more severe accumulation of ROS in chloroplasts in chilling-treated leaves than that in control leaves. Therefore, under chilling stress, the mitochondrial AOX pathway played an important role in protection of photosynthetic apparatus against photodamage, and this protective function was more efficient under chilling stress than in the control plants. However, the mechanisms of upregulation in AOX pathway under combined chilling and light stresses need further investigation.

\section{References}

Ara N., Naveedullah, Ding C. et al.: Inhibition of the alternative respiratory pathway at high temperatures leads to higher reactive oxygen species production and downregulation of the antioxidant defense system in squash leaves. - Plant Growth Regul. 79: 127-134, 2016.

Betti M., Bauwe H., Busch F.A. et al.: Manipulating photorespiration to increase plant productivity: recent advances and perspectives for crop improvement. - J. Exp. Bot. 67: 29772988, 2016.

Dahal K., Vanlerberghe G.C.: Alternative oxidase respiration 
maintains both mitochondrial and chloroplast function during drought. - New Phytol. 213: 560-571, 2017.

Dinakar C., Abhaypratap V., Yearla S.R. et al.: Importance of ROS and antioxidant system during the beneficial interactions of mitochondrial metabolism with photosynthetic carbon assimilation. - Planta 231: 461, 2010a.

Dinakar C., Raghavendra A.S., Padmasree K.: Importance of AOX pathway in optimizing photosynthesis under high light stress: role of pyruvate and malate in activating AOX. Physiol. Plantarum 139: 13-26, 2010b.

Dinakar C., Vishwakarma A., Raghavendra A.S., Padmasree K.: Alternative oxidase pathway optimizes photosynthesis during osmotic and temperature stress by regulating cellular ROS, malate valve and antioxidative systems. - Front. Plant Sci. 7: 68, 2016.

Dutilleul C., Driscoll S., Cornic G. et al.: Functional mitochondrial complex I is required by tobacco leaves for optimal photosynthetic performance in photorespiratory conditions and during transients. - Plant Physiol. 131: 264275, 2003.

Höglind M., Hanslin H.M., Mortensen L.M.: Photosynthesis of Lolium perenne L. at low temperatures under low irradiances.Environ. Exp. Bot. 70: 297-304, 2011.

Hu W.H., Yan X.H., He Y., Ye X.L.: Role of alternative oxidase pathway in protection against drought-induced photoinhibition in pepper leaves. - Photosynthetica 56: 1297-1303, 2018.

Juszczuk I.M., Ostaszewska M.: Respiratory activity, energy and redox status in sulphur-deficient bean plants. - Environ. Exp. Bot. 74: 245-254, 2011.

Murata N., Takahashi S., Nishiyama Y., Allakhverdiev S.I.: Photoinhibition of photosystem II under environmental stress. - BBA-Bioenergetics 1767: 414-421, 2007.

Murik O., Tirichine L., Prihoda J. et al.: Downregulation of mitochondrial alternative oxidase affects chloroplast function, redox status and stress response in a marine diatom. - New Phytol. 221: 1303-1316, 2019.

Nishiyama Y., Allakhverdiev S.I., Murata N.: A new paradigm for the action of reactive oxygen species in the photoinhibition of photosystem II. - BBA-Bioenergetics 1757: 742-749, 2006.

Nishiyama Y., Allakhverdiev S.I., Murata N.: Protein synthesis is the primary target of reactive oxygen species in the photoinhibition of photosystem II. - Physiol. Plantarum 142: 35-46, 2011.

Noguchi K., Yoshida K.: Interaction between photosynthesis and respiration in illuminated leaves. - Mitochondrion 8: 87-99, 2008.

Ogawa T., Sonoike K.: Dissection of respiration and photosynthesis in the cyanobacterium Synechocystis sp. PCC6803 by the analysis of chlorophyll fluorescence. - J. Photoch. Photobio. B 144: 61-67, 2015.

Patterson B.D., MacRae E.A., Ferguson I.B.: Estimation of hydrogen peroxide in plant extracts using titanium(IV). Anal. Biochem. 139: 487-492, 1984.

Raghavendra A.S., Padmasree K.: Beneficial interactions of mitochondrial metabolism with photosynthetic carbon assimilation. - Trends Plant Sci. 8: 546-553, 2003.

Scheibe R., Dietz K.J.: Reduction-oxidation network for flexible adjustment of cellular metabolism in photoautotrophic cells. Plant Cell Environ. 35: 202-216, 2012.

Strasser B.J., Strasser R.J.: Measuring fast fluorescence transients to address environmental questions: The JIP test. - In: Mathis P. (ed.): Photosynthesis: From Light to Biosphere. Vol. 5. Pp. 977-980. Kluwer Academic Publishers, Dordrecht 1995.

Strasser R.J., Srivastava A., Tsimilli-Michael M.: The fluorescence transient as a tool to characterize and screen photosynthetic samples. - In: Yunus M., Pathre U., Mohanty P. (ed.): Probing Photosynthesis: Mechanisms, Regulation and Adaptation. Pp. 445-483. Taylor \& Francis, London 2000.

Sunil B., Saini D., Bapatla R.B. et al.: Photorespiration is complemented by cyclic electron flow and the alternative oxidase pathway to optimize photosynthesis and protect against abiotic stress. - Photosynth. Res. 139: 67-79, 2019.

Vanlerberghe G.C.: Alternative oxidase: a mitochondrial respiratory pathway to maintain metabolic and signaling homeostasis during abiotic and biotic stress in plants. - Int. J. Mol. Sci. 14: 6805-6847, 2013.

Vishwakarma A., Tetali S.D., Selinski J. et al.: Importance of the alternative oxidase (AOX) pathway in regulating cellular redox and ROS homeostasis to optimize photosynthesis during restriction of the cytochrome oxidase pathway in Arabidopsis thaliana. - Ann. Bot.-London 116: 555-569, 2015.

Wang F., Wang X., Zhao C. et al.: Alternative pathway is involved in the tolerance of highland barley to the low-nitrogen stress by maintaining the cellular redox homeostasis. - Plant Cell Rep. 35: 317-328, 2016.

Yoshida K., Terashima I., Noguchi K.: Distinct roles of the cytochrome pathway and alternative oxidase in leaf photosynthesis. - Plant Cell Physiol. 47: 22-31, 2006.

Yoshida K., Terashima I., Noguchi K.: Up-regulation of mitochondrial alternative oxidase concomitant with chloroplast over-reduction by excess light. - Plant Cell Physiol. 48: 606614, 2007.

Zhang L.T., Gao H.Y., Zhang Z.S. et al.: Multiple effects of inhibition of mitochondrial alternative oxidase pathway on photosynthetic apparatus in Rumex K-1 leaves. - Biol. Plantarum 56: 365-368, 2012a.

Zhang L.T., Zhang Z.S., Gao H.Y. et al.: Mitochondrial alternative oxidase pathway protects plants against photoinhibition by alleviating inhibition of the repair of photodamaged PSII through preventing formation of reactive oxygen species in Rumex K-1 leaves. - Physiol. Plantarum 143: 396-407, 2011a.

Zhang L.T., Zhang Z.S., Gao H.Y. et al.: The mitochondrial alternative oxidase pathway protects the photosynthetic apparatus against photodamage in Rumex K-1 leaves. - BMC Plant Biol. 12: 40, 2012b.

Zhang Z.S., Jia Y.J., Gao H.Y. et al.: Characterization of PSI recovery after chilling-induced photoinhibition in cucumber (Cucumis sativus L.) leaves. - Planta 234: 883-889, 2011 b.

(C) The authors. This is an open access article distributed under the terms of the Creative Commons BY-NC-ND Licence. 